\title{
Promoter methylation analysis of IDH genes in human gliomas
}

\author{
Simon Flanagan ${ }^{1,2}$, Maggie Lee ${ }^{1,2}$, Cheryl C. Y. Li ${ }^{3}$, Catherine M. Suter ${ }^{3}$ and Michael E. Buckland ${ }^{1,2 *}$ \\ ${ }^{1}$ Discipline of Pathology, University of Sydney, Sydney, NSW, Australia \\ 2 Department of Neuropathology, Royal Prince Alfred Hospital, Sydney, NSW, Australia \\ ${ }^{3}$ Victor Chang Cardiac Research Institute, Darlinghurst, NSW, Australia
}

\section{Edited by:}

Lorenzo Galluzzi, Institut National de

la Santé et de la Recherche Medicale,

France

Reviewed by:

Xose S. Puente, Universidad de Oviedo, Spain

Laura E. Benjamin, Eli Lilly and Co., USA

${ }^{*}$ Correspondence:

Michael E. Buckland, Discipline of

Pathology, University of Sydney,

Room 502A, D06 Blackburn, NSW

2006, Australia.

e-mail:michael.buckland@

sydney.edu.au
Mutations in isocitrate dehydrogenase (IDH)-1 or -2 are found in the majority of WHO grade $\mathrm{II}$ and III astrocytomas and oligodendrogliomas, and secondary glioblastomas. Almost all described mutations are heterozygous missense mutations affecting a conserved arginine residue in the substrate binding site of IDH1 (R132) or IDH2 (R172). But the exact mechanism of IDH mutations in neoplasia is not understood. It has been proposed that IDH mutations impart a "toxic gain-of-function" to the mutant protein, however a dominantnegative effect of mutant IDH has also been described, implying that IDH may function as a tumor suppressor gene. As most, if not all, tumor suppressor genes are inactivated by epigenetic silencing, in a wide variety of tumors, we asked if IDH1 or IDH2 carry the epigenetic signature of a tumor suppressor by assessing cytosine methylation at their promoters. Methylation was quantified in 68 human brain tumors, including both IDH-mutant and IDH wildtype, by bisulfite pyrosequencing. In all tumors examined, CpG methylation levels were less than $8 \%$. Our data demonstrate that inactivation of IDH function through promoter hypermethylation is not common in human gliomas and other brain tumors. These findings do not support a tumor suppressor role for IDH genes in human gliomas.

Keywords: isocitrate dehydrogenase, glioma, brain tumor, DNA methylation, pyrosequencing

\section{INTRODUCTION}

Mutations in isocitrate dehydrogenase (IDH)-1 or-2 are found in the majority of WHO grade II and III astrocytomas and oligodendrogliomas, and secondary glioblastomas (Parsons et al., 2008; Yan et al., 2009). IDH mutations are also found in some myeloid malignancies and chondroid tumors (Amary et al., 2011). IDH mutations occur early in gliomagenesis (Watanabe et al., 2009), suggesting that low grade diffuse gliomas share a common pathway in their development. Patients with high-grade gliomas carrying IDH mutations have significantly better survival than those with wildtype IDH tumors and also respond better to treatment (Parsons et al., 2008; Yan et al., 2009; Houillier et al., 2010). In addition, IDH mutation status is useful in assisting diagnostic classification: the majority of astrocytomas, oligodendrogliomas, and secondary glioblastomas harbor IDH mutations while almost all primary glioblastomas do not (reviewed in Gupta et al., 2011).

Isocitrate dehydrogenase is one of several citric acid cycle enzymes implicated in neoplasia. Mutations in all four subunits of succinate dehydrogenase (SDH) underlie a variety of sporadic and inherited paragangliomas and pheochromocytomas (Sudarshan et al., 2007), while mutations in fumarate hydratase are associated with hereditary leiomyomatosis and renal cell cancer and some cases of isolated type 2 papillary renal cell carcinoma (Gardie et al., 2011). SDH and fumarate hydratase are thought to act as tumor suppressor genes, with cancers exhibiting the "two-hits" typical of tumor suppressor gene inactivation (reviewed in Wallace, 2012).
Almost all described IDH mutations in gliomas are heterozygous missense mutations focused on a few conserved residues in the enzymes' substrate binding sites. IDH mutations have thus been proposed to impart a toxic gain-of-function to the resultant protein (Dang et al., 2009). A salient biochemical feature of cells carrying mutant IDH is increased R-2 hydroxyglutarate $(2 \mathrm{HG})$ production via consumption of $\alpha \mathrm{KG}$ and NADPH (Dang et al., 2009). But mature IDH1 and IDH2 enzymes are homodimeric, and it has also been demonstrated that mutant IDH1 exerts a dominant-negative effect on IDH function through mutant/wildtype heterodimer formation (Zhao et al., 2009); this scenario would lead to a direct effect on $\alpha \mathrm{KG}$ and NADPH levels. We and others have previously reported loss of heterozygosity at the IDH1 locus in gliomas and leukemias (Ichimura et al., 2009; Zhang et al., 2011; Gupta et al., 2012), and monoallelic expression of IDH1 in gliomas is not uncommon (Walker et al., 2012). Furthermore a recent report characterizes several rare but recurrent IDH mutations that result in loss-of-function without elevation of 2HG (Ward et al., 2012). Taken together, these findings suggest that at least in some circumstances IDH1 and/or IDH2 may function as a typical tumor suppressor gene.

As promoter hypermethylation is one hallmark of tumor suppressor genes in a variety of tumors (Baylin and Herman, 2000), we asked if IDH genes may carry this particular epigenetic signature of a tumor suppressor by assessing cytosine methylation at their respective promoters. Our study is the first to specifically examine IDH promoter methylation in tumors. 


\section{MATERIALS AND METHODS TUMORS SAMPLES}

Tumors were obtained from the Royal Prince Alfred Hospital tumor and tissue bank following appropriate institutional human research ethics approval. Histological diagnoses were provided by an experienced neuropathologist (Michael E. Buckland). The tumor samples included gliomas with a variety of $\mathrm{IDH} 1$ mutations, as well as IDH-wildtype tumors (Table 1) and three samples of non-neoplastic brain. Also included in the group were two tumors with a proven IDH1 mutation, but with absent staining by the IDH1 mutationspecific antibodies H09 and SMab-1 (see below). All other tumors with IDH1 R132H or R132S mutations showed positive immunostaining with H09 or SMab-1 antibodies, respectively.

\section{IMMUNOHISTOCHEMISTRY}

Monoclonal antibodies against IDH1 R132H (clone H09; Dianova, Germany) and IDH1 R132S (kind gift from Dr. Y. Kato, Japan) were used at 1:500 dilution on $5 \mu \mathrm{m}$-FFPE tumor sections. Following antigen retrieval in $10 \mathrm{mM}$ sodium citrate buffer $\mathrm{pH} 6.0$, for $20 \mathrm{~min}$ at $125^{\circ} \mathrm{C}$, sections were incubated in primary antibodies for $1 \mathrm{~h}$ at room temperature, and antibody detection was performed using the Dako Envision system, according to the manufacturer's instructions.

\section{DNA EXTRACTION AND BISULFITE MODIFICATION}

DNA was extracted from $100 \mathrm{mg}$ of frozen tumor tissue using the Qiagen DNeasy blood and tissue kit (Qiagen, Hilden, Germany), and bisulfite modification was performed using the Qiagen Epitect Bisulfite Kit (Qiagen, Hilden, Germany), according to the manufacturer's instructions.

\section{PROMOTER METHYLATION ANALYSIS}

Methylation status of the $I D H 1$ and $I D H 2$ promoter regions were assessed using Qiagen's Pyromark CpG assays, Hs_IDH1_01_PM and Hs_IDH2_01_PM, respectively (see Figure 1). Pyrograms were analyzed using Pyromark Q24 software (Qiagen, Hilden, Germany), version 2.0.6, to calculate percentage methylation at each $\mathrm{CpG}$ and mean methylation across all CpGs for each sample was calculated.

Table 1 | Tumors tested, IDH1 mutation status, and mean methylation levels.

\begin{tabular}{lrlll}
\hline Diagnosis & N & $\begin{array}{l}\text { IDH1 } \\
\text { mutation }\end{array}$ & $\begin{array}{l}\text { Mean IDH1 } \\
\text { methylation } \\
\text { (\%) }\end{array}$ & $\begin{array}{l}\text { Mean IDH2 } \\
\text { methylation (\%) }\end{array}$ \\
\hline Glioblastoma & 30 & 8 & $1.7 \pm 0.9$ & $1.7 \pm 0.5$ \\
Astrocytoma & 7 & 6 & $2.6 \pm 0.8$ & $3.0 \pm 1.3$ \\
Oligodendroglioma & 10 & 9 & $2.2 \pm 0.7$ & $2.1 \pm 0.8$ \\
Pilocytic astrocytoma & 2 & 0 & $2.1 \pm 0.4$ & $1.9 \pm 0.0$ \\
Meningioma & 16 & 0 & $2.3 \pm 1.2$ & $3.4 \pm 1.6$ \\
Adenocarcinoma & 3 & 0 & $2.2 \pm 0.4$ & $2.6 \pm 0.9$ \\
Non-neoplastic brain & 3 & n/a & $3.0 \pm 1.75$ & $3.9 \pm 0.9$
\end{tabular}

\section{IDH1 AND IDH2 MUTATION ASSESSMENT}

In tumors that were negative for IDH R132H immunostaining, $I D H 1$ and $I D H 2$ mutation status was determined by direct DNA sequencing. The fourth exons of IDH1 and IDH2 were PCR amplified in separate reactions using primer pairs CATTTGTCTGAAAAACTTTGCTT and TCACATTATTGCCAACATGAC for IDH1, and GGTTCAAATTCTGGTTGAAAGATG and GCTAGGCGAGGAGCTCCAGT for IDH2. Each reaction consisted of $50 \mathrm{mM}$ Tris/ $\mathrm{HCl}, 10 \mathrm{mM} \mathrm{KCl}, 5 \mathrm{mM}\left(\mathrm{NH}_{4}\right)_{2} \mathrm{SO}_{4}, 2 \mathrm{mM} \mathrm{MgCl}_{2}$, $0.2 \mathrm{mM}$ each dNTP, $2 \mathrm{U}$ FastStart Taq polymerase (Roche, Manheim, Germany), $0.5 \mu \mathrm{M}$ each primer, and $2 \mu \mathrm{L}$ of extracted DNA in a total volume of $20 \mu \mathrm{L}$. Cycling conditions were, initial denaturation at $95^{\circ} \mathrm{C}$ for $5 \mathrm{~min}$, followed by 40 cycles of $95^{\circ} \mathrm{C}$ for $30 \mathrm{~s}, 60^{\circ} \mathrm{C}$ for $30 \mathrm{~s}$, and $72^{\circ} \mathrm{C}$ for $30 \mathrm{~s}$. PCR products were sequenced directionally using BigDye Terminator v3.1 Cycle Sequencing Kit (Applied Biosystems), and sequencing reaction products were resolved on a 3730xl DNA Analyzer (Applied Biosystems), according to the manufacturer's instructions.

\section{STATISTICS}

Two-tailed Student $t$-test was used to compare mean IDH1 and IDH2 promoter methylation levels between IDH-mutant and wildtype tumors.

\section{RESULTS}

Figure 1 shows the promoter regions of $I D H 1$ and $I D H 2$, the $\mathrm{CpG}$ island within these regions, and the sequences targeted for bisulfite pyrosequencing. The $\mathrm{IDH} 1$ assay targets four contiguous $\mathrm{CpG}$ sites, $275 \mathrm{bp}$ upstream of the transcription start site. The IDH2 assay targets eight $\mathrm{CpG}$ sites $425 \mathrm{bp}$ upstream from the transcription start site. The CpGs targeted by these assays lie within $\mathrm{CpG}$ islands that are adjacent to, or span, the transcription start site of the gene. Typical pyrograms obtained for patient samples for both $I D H 1$ and $I D H 2$ assays are shown in Figure 2.

A total of 68 brain tumors were assessed for IDH1 and IDH2 promoter methylation status. Tumors examined consisted of 49 gliomas, 16 meningiomas, and 3 metastatic adenocarcinomas. Twenty five $I D H 1$ mutations were present in the gliomas tested $(23 \times \mathrm{R} 132 \mathrm{H}, 1 \times \mathrm{R} 132 \mathrm{~S}$, and $1 \times \mathrm{R} 132 \mathrm{~L})$; no IDH2-mutant tumors were present. Two of the mutant tumors $(1 \times \mathrm{R} 132 \mathrm{H}$ and $1 \times$ R132S) were consistently negative for mutant protein expression on immunohistochemistry; all other R132H-mutant tumors were immunopositive with $\mathrm{H} 09$ antibody. Table 1 shows the number of tumor types examined, their IDH1 mutation status, and mean promoter methylation levels for $I D H 1$ and $I D H 2$. Table A1 in Appendix details the tumor type, WHO grade, IDH mutation status, and the methylation levels at each individual $\mathrm{CpG}$ dinucleotide for all samples included in this study.

For $I D H 1$, mean of methylation across all tumors was $2 \%$ (below the reported detection limit of pyrosequencing; Mikeska et al., 2011), with average methylation across the four CpGs assayed for each sample ranging from 0.3 to $5.3 \%$. The highest methylation level detected (5.3\%) was in a secondary glioblastoma that harbored an IDH1 R132H mutation. No other samples in the study showed methylation above 5\% at the IDH1 promoter (Table A1 in Appendix). The three nonneoplastic brain samples had an average methylation level of 


\section{IDH1}

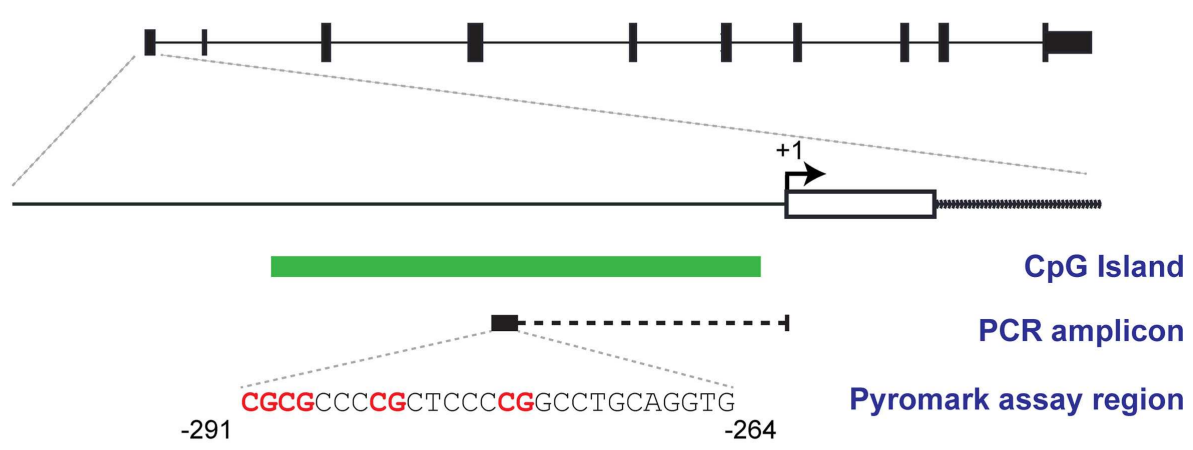

IDH2
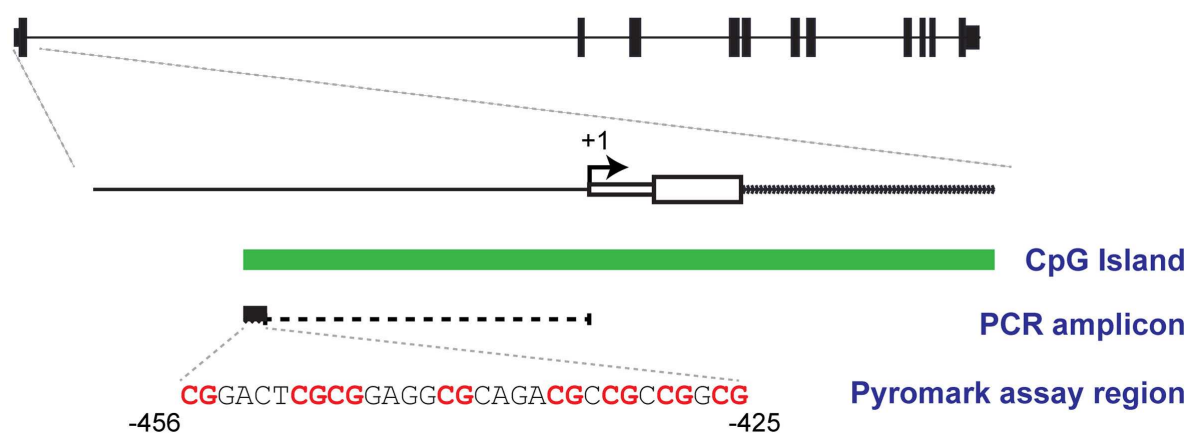

FIGURE 1 | Bisulphite Pyrosequencing designs. Schematics showing regions targeted for methylation analysis and their relationships with CpG islands and transcription start sites of $I D H 1$ and $I D H 2$.

$3 \%$. There was no significant difference in mean methylation levels between wildtype tumors and those harboring an IDH1 mutation.

For $I D H 2$, average methylation across all tumor samples was $2.3 \%$, and the highest average methylation across the eight CpGs assayed shown by any sample was $7.1 \%$ in a meningioma (Table A1 in Appendix). Three non-neoplastic control brain samples had an average methylation level of 3.9\%. Only one other sample, also a meningioma, recorded an average methylation at the IDH2 promoter above 5\%. In vitro methylated genomic DNA (Qiagen, Hilden, Germany) showed average methylation of 85.2 and $87.7 \%$ for the $I D H 1$ and $I D H 2$ loci, respectively, confirming assay performance.

Two gliomas in our cohort were found to harbor IDH1 mutations when subjected to DNA sequencing, but exhibited no protein expression by immunohistochemistry, despite repeated testing. Following negative immunohistochemistry results, we were able to extract DNA from the slide used for immunostaining, and confirm the presence of $I D H 1$ mutation, excluding tissue mosaicism as a cause of these results. The two IDH-mutant tumors without detectable mutant protein expression showed mean IDH1 methylation levels of $2.8 \%(\mathrm{R} 132 \mathrm{H})$, and $1.8 \%$ (R132S) only.

\section{DISCUSSION}

In this study, we quantified methylation levels within the $\mathrm{CpG}$ island associated with the promoter regions of both $I D H 1$ and $\mathrm{IDH} 2$ by bisulfite pyrosequencing. We hypothesized that if $\mathrm{IDH} 1$ and $I D H 2$ functioned as tumor suppressor genes, then some tumors may exhibit dense hypermethylation of the $\mathrm{CpG}$ island associated with their promoter region. Loss of gene expression associated with promoter methylation is a well described phenomenon in a variety of tumors (Baylin and Herman, 2000). However, none of the 68 tumors examined exhibited this epigenetic hallmark of tumor suppressor genes.

Three IDH enzymes are present in mammalian cells, IDH1, IDH2, and IDH3. IDH3 utilizes NAD+ as the reducing agent in an irreversible reaction to convert mitochondrial isocitrate to $\alpha \mathrm{KG}$, while both IDH1 and IDH2 utilize NADP+ to convert isocitrate to $\alpha \mathrm{KG}$ in a reversible fashion. To date, IDH3 mutations have not been implicated in neoplasia. Mutant IDH1 and IDH2 enzymes exert a novel biochemical effect, namely the production of $2 \mathrm{HG}$, via consumption of $\alpha \mathrm{KG}$ and NADPH (Dang et al., 2009). $2 \mathrm{HG}$ is a competitive inhibitor of $\alpha \mathrm{KG}$-dependant enzymes such as histone lysine methyltransferases and the TET family of 5-methylcytosine hydroxylases. Both reduced $\alpha \mathrm{KG}$ and increased $2 \mathrm{HG}$ levels converge to alter the cellular epigenetic 


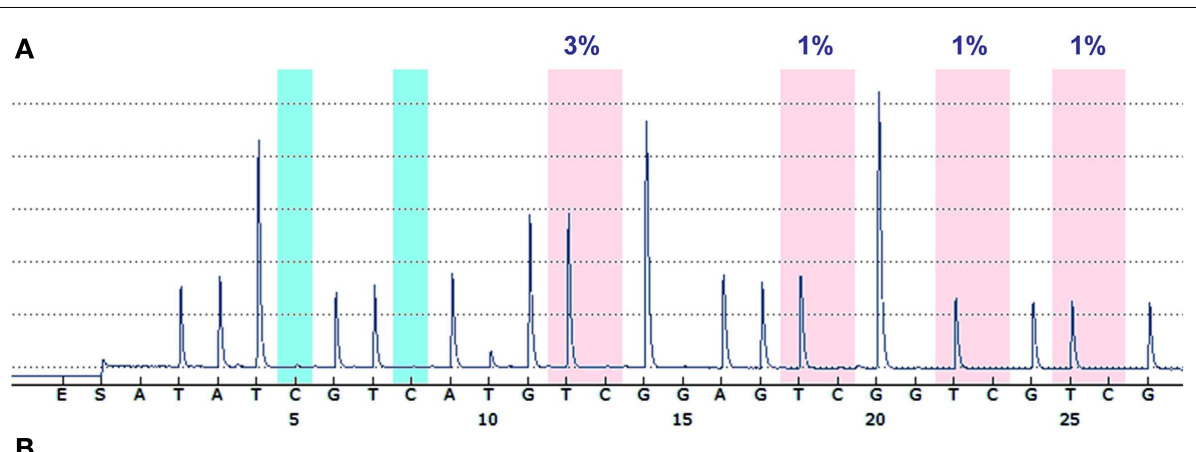

$\mathbf{B}$

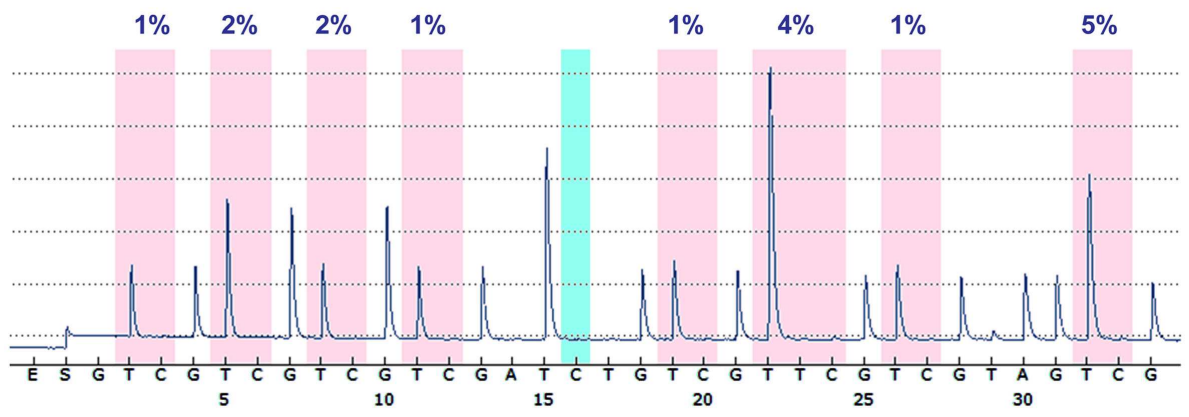

FIGURE 2 | Representative pyrograms. Typical pyrograms obtained from patient samples for IDH1 (A) and IDH2 (B). Each pink shaded column indicates a $\mathrm{CpG}$ dinucleotide assayed, and the percentage of methylation at that $\mathrm{CpG}$ dinucleotide is indicated above. The blue shaded boxes are internal bisulfite modification control assessments.

landscape; in vitro transfection of mutant IDH into immortalized astrocytes results in increased histone methylation and a gradual rise in DNA methylation levels (Lu et al., 2012). In addition, the recently described glioma CpG island hypermethylator phenotype is associated with IDH mutations (Turcan et al., 2012).

IDH1 is also the major source of non-mitochondrial NADPH in humans, which is important in the control of oxidative damage. Either loss-of-function or gain-of-function of IDH will result in reduced cytoplasmic NADPH levels and susceptibility to oxidative stress (Reitman and Yan, 2010). It may be that neomorphic production of $2 \mathrm{HG}$ and decreased enzymatic activity are both involved in tumor development, but in different contexts. In support of this, rare IDH mutations in lymphoma, pediatric glioblastoma, and thyroid cancer have demonstrated loss-of-function effects (Ward et al., 2012). Assessment of IDH promoter methylation in these tumor types may assist in determining if tissue-specific roles exist for IDH mutations in cancer.

Monoallelic expression of $I D H 1$ has recently been reported in 12 out of 67 gliomas with IDH1 mutations (Walker et al., 2012), with expression occurring only from the wildtype allele in 10 of 12 cases. Preferential or exclusive expression of the wildtype IDH1 allele was associated with significantly poorer outcomes (Walker et al., 2012). Included in our cohort were two tumors harboring IDH1 mutations but without detectable mutant protein expression by immunohistochemistry, raising the possibility of monoallelic expression from the wildtype allele. However, neither of these tumors showed significant promoter methylation, excluding this epigenetic regulatory mechanism as the cause of undetectable mutant protein. Further studies into the mechanism(s) involved in monoallelic expression and its role in progression of glioma and other cancers will provide additional insights into the role of IDH in neoplasia.

Molecular testing for IDH mutations is useful in the clinical setting in assisting diagnostic classification and as a prognostic indicator. While there is a routinely used, specific antibody for IDH1 $\mathrm{R} 132 \mathrm{H}$ mutations, it is becoming common to screen tumors with negative IDH immunostaining using DNA based techniques. The identification of monoallelic expression of IDH1 in glioma raises potential issues in the clinical interpretation of these molecular analyses. It may be that monoallelic (or even skewed) gene expression could compromise the prognostic utility of DNA testing for a subset of tumors.

In this study, we have demonstrated that promoter hypermethylation of $I D H 1$ and $I D H 2$ genes is absent or very rare in human gliomas and other brain tumors. These findings do not support a tumor suppressor gene mechanism for IDH in human gliomas. However the occurrence of uncommon inactivating IDH mutations, loss of heterozygosity of IDH in some tumors, and monoallelic expression of IDH1 in some gliomas points to as yet uncharacterized mechanisms of disease associated with IDH dysfunction.

\section{ACKNOWLEDGMENT}

This work was supported by the Cancer Institute NSW and the Sydney Foundation for Medical Research. 


\section{REFERENCES}

Amary, M. F., Bacsi, K., Maggiani, F., Damato, S., Halai, D., Berisha, F., et al. (2011). IDH1 and IDH2 mutations are frequent events in central chondrosarcoma and central and periosteal chondromas but not in other mesenchymal tumours. J. Pathol. 224, 334-343.

Baylin, S. B., and Herman, J. G. (2000). DNA hypermethylation in tumorigenesis: epigenetics joins genetics. Trends Genet. 16, 168-174.

Dang, L., White, D. W., Gross, S., Bennett, B. D., Bittinger, M. A., Driggers, E. M., et al. (2009). Cancer-associated IDH1 mutations produce 2-hydroxyglutarate. Nature 462, 739-744.

Gardie, B., Remenieras, A., Kattygnarath, D., Bombled, J., Lefevre, S., Perrier-Trudova, V., et al. (2011). Novel FH mutations in families with hereditary leiomyomatosis and renal cell cancer (HLRCC) and patients with isolated type 2 papillary renal cell carcinoma. J. Med. Genet. 48, 226-234.

Gupta, R., Flanagan, S., Lee, M., Shivalingham, B., Malaki, S., Wheeler, H. R., et al. (2012). Expanding the spectrum of IDH mutations in gliomas. Mod. Pathol. (in press).

Gupta, R., Webb-Myers, R., Flanagan, S., and Buckland, M. E. (2011). Isocitrate dehydrogenase mutations in diffuse gliomas: clinical and aetiological implications. J. Clin. Pathol. 64, 835-844.
Houillier, C., Wang, X., Kaloshi, G., Mokhtari, K., Guillevin, R., Laffaire, J., et al. (2010). IDH1 or IDH2 mutations predict longer survival and response to temozolomide in low-grade gliomas. Neurology 75, 1560-1566.

Ichimura, K., Pearson, D. M., Kocialkowski, S., Backlund, L. M., Chan, R., Jones, D. T., et al. (2009). IDH1 mutations are present in the majority of common adult gliomas but rare in primary glioblastomas. Neurooncology 11, 341-347.

Lu, C., Ward, P. S., Kapoor, G. S., Rohle, D., Turcan, S., AbdelWahab, O., et al. (2012). IDH mutation impairs histone demethylation and results in a block to cell differentiation. Nature 483, 474-478.

Mikeska, T., Felsberg, J., Hewitt, C. A., and Dobrovic, A. (2011). Analysing DNA methylation using bisulphite pyrosequencing. Methods Mol. Biol. 791, 33-53.

Parsons, D. W., Jones, S., Zhang, X., Lin, J. C., Leary, R. J., Angenendt, P., et al. (2008). An integrated genomic analysis of human glioblastoma multiforme. Science 321, 1807-1812.

Reitman, Z. J., and Yan, H. (2010). Isocitrate dehydrogenase 1 and 2 mutations in cancer: alterations at a crossroads of cellular metabolism. J. Natl. Cancer Inst. 102, 932-941.

Sudarshan, S., Pinto, P. A., Neckers, L., and Linehan, W. M. (2007).
Mechanisms of Disease: hereditary leiomyomatosis and renal cell cancer - a distinct form of hereditary kidney cancer. Nat. Clin. Pract. Urol. 4, 104-110.

Turcan, S., Rohle, D., Goenka, A., Walsh, L. A., Fang, F., Yilmaz, E., et al. (2012). IDH1 mutation is sufficient to establish the glioma hypermethylator phenotype. Nature 483 479-483.

Walker, E. J., Zhang, C., CasteloBranco, P., Hawkins, C., Wilson, W., Zhukova, N., et al. (2012). Monoallelic expression determines oncogenic progression and outcome in benign and malignant brain tumors. Cancer Res. 72, 636-644.

Wallace, D. C. (2012). Mitochondria and cancer. Nat. Rev. Cancer 12, 685-698.

Ward, P. S., Cross, J. R., Lu, C., Weigert, O., Abel-Wahab, O., Levine, R. L., et al. (2012). Identification of additional IDH mutations associated with oncometabolite $\quad \mathrm{R}(-)$-2-hydroxyglutarate production. Oncogene 31 2491-2498.

Watanabe, T., Nobusawa, S., Kleihues, P., and Ohgaki, H. (2009). IDH1 mutations are early events in the development of astrocytomas and oligodendrogliomas. Am. J. Pathol. 174, 1149-1153.

Yan, H., Parsons, D. W., Jin, G., Mclendon, R., Rasheed, B. A., Yuan, W., et al. (2009). IDH1 and IDH2 mutations in gliomas. N. Engl. J. Med. 360, 765-773.
Zhang, Y., Wei, H., Wang, M., Huai, L., Mi, Y., Lin, D., et al. (2011). Some novel features of IDH1-mutated acute myeloid leukemia revealed in Chinese patients. Leuk. Res. 35, 1301-1306.

Zhao, S., Lin, Y., Xu, W., Jiang, W., Zha, Z., Wang, P., et al. (2009). Gliomaderived mutations in IDH1 dominantly inhibit IDH1 catalytic activity and induce HIF-1alpha. Science 324, 261-265.

Conflict of Interest Statement: The authors declare that the research was conducted in the absence of any commercial or financial relationships that could be construed as a potential conflict of interest.

Received: 02 October 2012; accepted: 29 November 2012; published online: 19 December 2012.

Citation: Flanagan S, Lee $M, L i C C Y$, Suter CM and Buckland ME (2012) Promoter methylation analysis of IDH genes in human gliomas. Front. Oncol. 2:193. doi: 10.3389/fonc.2012.00193

This article was submitted to Frontiers in Molecular and Cellular Oncology, a specialty of Frontiers in Oncology.

Copyright (c) 2012 Flanagan, Lee, Li, Suter and Buckland. This is an openaccess article distributed under the terms of the Creative Commons Attribution License, which permits use, distribution and reproduction in other forums, provided the original authors and source are credited and subject to any copyright notices concerning any third-party graphics etc. 


\section{APPENDIX}

Table A1 | Detailed results of tumours tested.

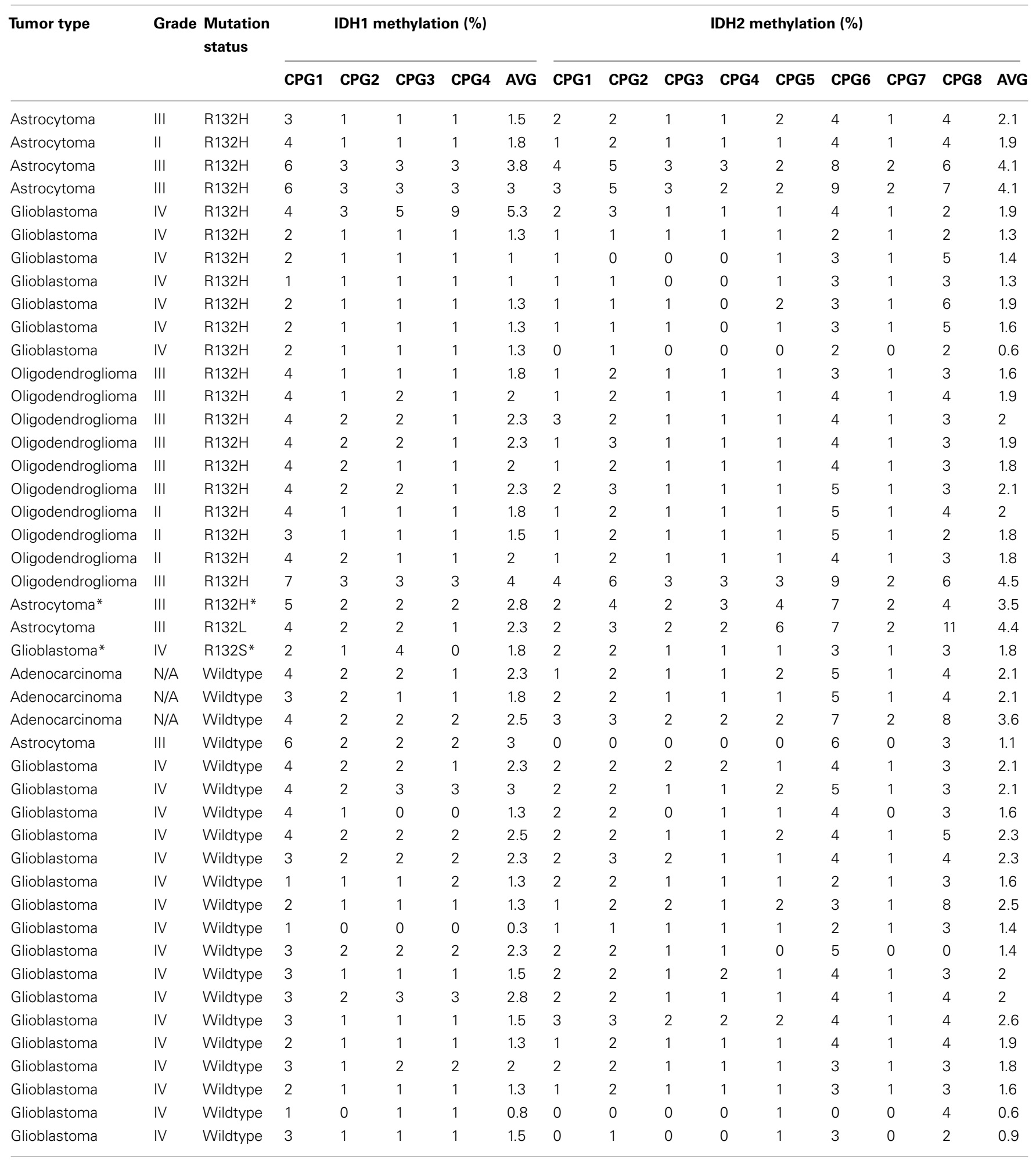


Table A1 | Continued

\begin{tabular}{|c|c|c|c|c|c|c|c|c|c|c|c|c|c|c|c|c|}
\hline \multirow[t]{2}{*}{ Tumor type } & \multirow[t]{2}{*}{ Grade } & \multirow{2}{*}{$\begin{array}{l}\text { Mutation } \\
\text { status }\end{array}$} & \multicolumn{5}{|c|}{ IDH1 methylation (\%) } & \multicolumn{9}{|c|}{ IDH2 methylation (\%) } \\
\hline & & & CPG1 & CPG2 & CPG3 & CPG4 & AVG & CPG1 & CPG2 & CPG3 & CPG4 & CPG5 & CPG6 & CPG7 & CPG8 & AVG \\
\hline Glioblastoma & IV & Wildtype & 1 & 0 & 1 & 1 & 0.8 & 1 & 1 & 0 & 1 & 2 & 3 & 1 & 3 & 1.5 \\
\hline Glioblastoma & IV & Wildtype & 2 & 1 & 1 & 1 & 1.3 & 0 & 0 & 0 & 0 & 1 & 3 & 0 & 5 & 1.1 \\
\hline Glioblastoma & IV & Wildtype & 2 & 1 & 1 & 1 & 1.3 & 1 & 2 & 1 & 1 & 1 & 4 & 1 & 4 & 1.9 \\
\hline Meningioma & 1 & Wildtype & 4 & 1 & 2 & 1 & 2 & 3 & 2 & 3 & 2 & 5 & 8 & 4 & 30 & 7.1 \\
\hline Meningioma & 1 & Wildtype & 3 & 2 & 1 & 1 & 1.8 & 2 & 2 & 2 & 1 & 4 & 7 & 2 & 12 & 4 \\
\hline Meningioma & I & Wildtype & 4 & 2 & 1 & 1 & 2 & 2 & 3 & 1 & 1 & 2 & 4 & 1 & 5 & 2.4 \\
\hline Meningioma & 1 & Wildtype & 3 & 1 & 1 & 1 & 1.5 & 1 & 2 & 1 & 1 & 1 & 5 & 1 & 4 & 2 \\
\hline Meningioma & 1 & Wildtype & 4 & 2 & 2 & 1 & 2.3 & 1 & 3 & 1 & 1 & 2 & 5 & 1 & 5 & 2.4 \\
\hline Meningioma & II & Wildtype & 3 & 1 & 1 & 1 & 1.5 & 1 & 3 & 0 & 1 & 1 & 4 & 1 & 5 & 2 \\
\hline Meningioma & I & Wildtype & 2 & 1 & 1 & 1 & 1.3 & 1 & 2 & 2 & 1 & 1 & 4 & 1 & 5 & 2.1 \\
\hline Meningioma & 1 & Wildtype & 2 & 1 & 1 & 1 & 1.3 & 0 & 1 & 1 & 0 & 1 & 3 & 0 & 4 & 1.3 \\
\hline Meningioma & 1 & Wildtype & 6 & 3 & 3 & 3 & 3.8 & 3 & 6 & 3 & 3 & 3 & 9 & 2 & 8 & 4.6 \\
\hline Meningioma & ॥ & Wildtype & 7 & 3 & 4 & 2 & 4 & 4 & 6 & 3 & 3 & 2 & 10 & 3 & 8 & 4.9 \\
\hline Meningioma & 1 & Wildtype & 7 & 3 & 3 & 3 & 4 & 3 & 6 & 3 & 2 & 3 & 9 & 2 & 9 & 4.6 \\
\hline Meningioma & II & Wildtype & 7 & 3 & 3 & 3 & 4 & 4 & 5 & 3 & 3 & 3 & 10 & 3 & 11 & 5.3 \\
\hline Meningioma & 1 & Wildtype & 5 & 3 & 3 & 3 & 3.5 & 2 & 4 & 2 & 2 & 2 & 8 & 1 & 7 & 3.5 \\
\hline Pilocytic astrocytoma & I & Wildtype & 4 & 2 & 2 & 1 & 2.3 & 2 & 2 & 1 & 1 & 1 & 4 & 1 & 3 & 1.9 \\
\hline Pilocytic astrocytoma & I & Wildtype & 3 & 1 & 2 & 1 & 1.8 & 1 & 1 & 2 & 1 & 1 & 4 & 1 & 4 & 1.9 \\
\hline \multicolumn{3}{|c|}{$t$-Test WTvs MUT (all tumors) } & 0.155 & 0.230 & 0.162 & 0.242 & 0.194 & 0.448 & 0.319 & 0.387 & 0.391 & 0.431 & 0.414 & 0.436 & 0.090 & 0.298 \\
\hline
\end{tabular}

Methylation is reported as a percentage of methylated alleles. Mutated tumours with absent mutant protein are marked by * 